\title{
LISTADO ACTUALIZADO Y CLAVES PARA POLYPLACOPHORA (MOLLUSCA) EN CUBA
}

\author{
Carmen R. Tejeda ${ }^{1}$, David Maceira ${ }^{1}$, Cedar García-Ríos ${ }^{2}$ y José Espinosa ${ }^{3}$ \\ ${ }^{1}$ Centro Oriental de Ecosistemas y Biodiversidad (BIOECO). Enramadas, No.601, esquina Barnada. \\ Santiago de Cuba, 90100. Cuba. carmenrosa@bioeco.ciges.inf.cu, david@bioeco.ciges.inf.cu \\ ${ }^{2}$ Departamento de Biología, Universidad de Puerto Rico en Humacao, Humacao, Puerto Rico 00791. \\ cedar.uprh@gmail.com \\ ${ }^{3}$ Instituto de Oceanología, Avenida $1^{\text {ra }}$ esquina a 186, No. 18406, La Habana, CP. 11600, Cuba. \\ espinosa@oceano.inf.cu
}

\section{RESUMEN}

Actualmente existe un parcial desconocimiento sobre cuáles especies de quitones están presentes en Cuba y sobre su correcto arreglo taxonómico, además, se carece de claves dicotómicas para su identificación. Empleando el listado de los moluscos marinos cubanos elaborado por Espinosa (2006), se actualizó la clase Polyplacophora empleando los criterios taxonómicos de García-Ríos (2003), Espinosa et al. (2012) y de Kaas y Van Belle (1998). Esta actualización permitió registrar por primera vez para Cuba un orden, dos subórdenes, cuatro familias, siete subfamilias, 12 géneros y 27 especies de quitones vivientes y se proponen claves que permiten la identificación de los taxones.

Palabras clave: Polyplacophora, quitones vivientes, claves dicotómicas, listado taxonómico, Cuba.

Title: Updated list and keys to Polyplacophora (Mollusca) from Cuba.

\section{ABSTRACT}

Currently, there is a lack of precise knowledge about which species of chitons occur in Cuba and their correct taxonomic arrangement, moreover, no dichotomous keys for identifying the taxa are available. From the Cuban marine mollusks list by Espinosa (2006), the class Polyplacophora was updated using the taxonomic arrangement by García-Ríos (2003), Espinosa et al. (2012) and Kaas and Van Belle (1998). This update allowed recording for the first time in Cuba one order, two suborders, four families, seven subfamilies, 12 genera and 27 species of living chitons and several dichotomous keys are proposed for the taxa identification.

Keywords: Polyplacophora, living chitons, dichotomous keys, taxonomic list, Cuba.

\section{INTRODUCCIÓN}

Los moluscos marinos cubanos comprenden 1545 especies (1 123 gasterópodos, 320 bivalvos, 39 escafópodos, 36 cefalópodos, 27 poliplacóforos y un aplacóforo), la gran mayoría de las cuales (1 162), se distribuyen por las zonas nerítica y litoral, mientras que para la zona circalitoral y el sistema afital o profundo se registran 323 y unas 60 especies tienen hábitos de vida pelágicos. Cuba constituye la localidad tipo de 287 especies de moluscos marinos, 249 gastrópodos, 27 bivalvos, 8 escafópodos y 3 cefalópodos (Espinosa, 2006).

En relación con la Clase Polyplacophora se conocen unas 850 especies recientes de quitones (Slieker, 2000), la mayoría de las cuales viven en el Pacífico occidental y en la costa occidental de América Central, mientras que en el Caribe y el Mediterráneo europeo están más pobremente representados (Lyons y Moretzsohn, 2009). 
Recientemente, Espinosa et al. (2012) aclaran que Lyons y Moretzsohn (2009) citaron 28 especies para Cuba, dos más de las 26 señaladas por Espinosa et al. (1995), con la adición de Stenoplax floridana (Pilsbry) y Americhiton andersoni (Watters). Estos autores confirmaron la presencia de esta última especie, no así la de $S$. floridana cuyo registro por Aguayo y Jaume (1936) se basó en dos valvas sueltas de ejemplares juveniles, recolectadas frente a Varadero, Matanzas, el cual fue desestimado por Espinosa y Cruz (1985) y lo incorporaron a la sinonimia de $S$. purpurascens (C. B. Adam).

En el último arreglo para la clase Polyplacophora se reconocen un orden, dos subórdenes, tres familias, seis subfamilias, 12 géneros y 27 especies para Cuba (Espinosa, 2006); pero nuevas consideraciones estiman un número diferente de táxones presentes en Cuba.

\section{OBJETIVO}

- Esclarecer el estatus de las especies de Polyplacophora presentes en Cuba y proponer claves dicotómicas para su identificación.

\section{MATERIALES Y MÉTODOS}

Se actualizó el listado taxonómico para la Clase Polyplacophora en Cuba a partir de la propuesta hecha por Espinosa (2006) empleando los criterios taxonómicos de García-Ríos (2003), Espinosa et al. (2012) y de Kaas y Van Belle (1998).

Las claves para los géneros Ischnochiton Gray, Stenoplax Carpenter in Dall, y Lepidochitona Gray, corresponden a la propuesta por Kaas, 1972: 66-68; y para lo géneros Acanthochitona Gray y Chiton Linné, también son propuestas por Kaas, 1972: 37-38 y 101-102, respectivamente. Todas las claves fueron modificadas según criterios actualizados de García-Ríos (2003). La clave para separar las familias y las claves para los géneros y las especies de Ischnochitonidae, Chitonidae (excepto Chiton) y los géneros de Acanthochitonidae son propuestas de este estudio. La clave para separar las especies de Stenoplax está basada en Kaas y Van Belle (1987) y la clave para separar las especies de Acanthochitona está basada en Lyons (1988).

\section{RESULTADOS Y DISCUSIÓN}

Se obtuvo un listado taxonómico actualizado para la clase Polyplacophora en Cuba conformado por un orden, dos subórdenes, cuatro familias, siete subfamilias, 12 géneros y 27 especies (Tabla I). En relación con los datos publicados por Espinosa (2006), el actual arreglo taxonómico posee algunos cambios que se detallan a continuación.

La familia Ischnochitonidae estuvo conformada por la subfamilia Ischnochitoninae sensu Espinosa (2006) y Espinosa et al. (2012), en el presente arreglo taxonómico se adicionan las subfamilias Callistoplacinae y Lepidochitoninae, antes consideradas como familias sensu Espinosa (2006) y Espinosa et al. (2012).

La actual subfamilia Callistoplacinae, antes considerada a nivel de familia, estuvo compuesta sólo por el género Callistochiton Dall, 1879, ahora se adicionan los géneros Ischnoplax Carpenter in Dall, 1879 y Ceratozona Dall, 1882. Este último género antes se ubicó en la familia Mopalidae, la cual queda sin representantes para Cuba. La subfamilia Lepidochitoninae mantiene el mismo género Lepidochitona Gray, 1821 que cuando se consideró familia. 
La familia Acanthochitonidae se conformó por cuatro géneros: Acanthochitona Gray, 1821, Americhiton Watters, 1990, Cryptoconchus Von Middendorff, 1847 y Choneplax Carpenter in Dall, 1882, sensu Espinosa (2006) y Espinosa et al. (2012). Actualmente se conforma sólo por Acanthochitona y Cryptoconchus. El género Americhiton no se reconoce para Cuba y su anterior especie registrada queda como Acanthochitona andersoni (Watters). El género Choneplax, con su única especie $C$. lata (Guilding, 1829), se ubica ahora en la familia Cryptoplacidae, registrada para Cuba.

Tabla I. Listado taxonómico actualizado de Polyplacophora para Cuba.

\begin{tabular}{|c|}
\hline Clase POLYPLACOPHORA Gray, 1821 \\
\hline Orden NEOLORICATA Bergenhayn, 1955 \\
\hline Suborden ISCHNOCHITONINA Bergenhayn, 1930 \\
\hline Familia Ischnochitonidae Dall, 1889 \\
\hline Subfamilia Ischnochitoninae s. s \\
\hline Género Ischnochiton Gray, 1847 \\
\hline Ischnochiton erythronotus (C. B. Adams, 1845) \\
\hline Ischnochiton papillosus (C. B. Adams, 1845) \\
\hline Ischnochiton striolatus (Gray, 1828) \\
\hline Género Stenoplax Carpenter in Dall, 1879 \\
\hline Stenoplax bahamensis Kaas y Belle, 1987 \\
\hline Stenoplax purpurascens (C. B. Adams, 1845) \\
\hline Stenoplax boogii (Haddon, 1886) \\
\hline Subfamilia Callistoplacinae Pilsbry, 1893 \\
\hline Género Ceratozona Dall, 1882 \\
\hline Ceratozonasqualida (C. B. Adams, 1845) \\
\hline Género Ischnoplax Carpenter in Dall, 1879 \\
\hline Ischnoplax pectinata (Sowerby, 1840) \\
\hline Género Callistochiton Dall, 1879 \\
\hline Callistochiton shuttleworthianus Pilsbry, 1893 \\
\hline Subfamilia Lepidochitoninae Iredale, 1914 \\
\hline Género Lepidochitona Gray, 1821 \\
\hline Lepidochitona liozonis (Dall y Simpson, 1901) \\
\hline Lepidochitona rosea Kaas, 1972 \\
\hline Familia Chitonidae Rafinisque, 1815 \\
\hline Subfamilia Chitoninae Rafinisque , 1815 \\
\hline Género Chiton Linné, 1758 \\
\hline Chiton marmoratus Gmelin, 1791 \\
\hline Chiton squamosus Linné, 1764 \\
\hline Chiton tuberculatus Linné, 1758 \\
\hline Chiton viridis Spengler, 1797 \\
\hline Subfamilia Acanthopleurinae Dall, 1889 \\
\hline Género Acanthopleura Guilding, 1829 \\
\hline
\end{tabular}




\begin{tabular}{|l|}
\hline Acanthopleura granulata (Gmelin,1791) \\
\hline Subfamilia Toniciinae Pilsbry, 1893 \\
\hline Género Tonicia Gray, 1847 \\
\hline Tonicia schrammi (Suttleworth, 1856) \\
\hline Suborden ACANTHOCHITONINA Bergenhayn, 1930 \\
\hline Familia Acanthochitonidae Pilsbry, 1893 \\
\hline Subfamilia Acanthochitoninae s.s. \\
\hline Género Acanthochitona Gray, 1821 \\
\hline Acanthochitona andersoni Watters, 1981 \\
\hline Acanthochitona astrigera (Reeve, 1847) \\
\hline Acanthochitona balesae Pilsbryin Abbott, 1954 \\
\hline Acanthochitona hemphilli (Pilsbry, 1893) \\
\hline Acanthochitona lineata Lyons, 1988 \\
\hline Acanthochitona pygmaea (Pilsbry, 1893) \\
\hline Acanthochitona roseojugum Lyons, 1988 \\
\hline Acanthochitona zebra Lyons, 1988 \\
\hline Género Cryptoconchus von Middendorff, 1847 \\
\hline Cryptoconchus floridanus (Dall, 1889) \\
\hline Familia Cryptoplacidae H. y A. Adams, 1858 \\
\hline Género Choneplax Carpenter in Dall, 1882 \\
\hline Choneplax lata (Guilding, 1829) \\
\hline
\end{tabular}

\section{CLAVES DICOTÓMICAS PARA LA CLASE POLYPLACOPHORA EN CUBA}

\section{CLAVE PARA FAMILIAS}

1a. Presencia de18 mechones con espículas en el dorso del cinturón

1b. Diferente al anterior

2a. Mechones suturales muy cortos, valva anal sin hendiduras.

Crypthoplacidae (Choneplax lata)

2b.Con dos o más hendiduras en la valva anal Acanthochitonidae

3a. Placa de inserción pectinada Chitonidae

3b. Placa de inserción no pectinada Ischnochitonidae 


\section{CLAVE PARA GÉNEROS DE ISCHNOCHITONIDAE DALL}

1a. Cinturón cubierto dorsalmente por escamas estriadas ............................................... 2

1b. Cinturón no está cubierto dorsalmente por escamas .................................................. 5

2a. Presencia de dos tipos de escamas en el dorso del cinturón, unas muy grandes entre otras de menor tamaño. género Ischnoplax, I. pectinata

2b. Escamas del dorso del cinturón de tamaños similares 3

3a. Animal alargado, el ancho es aproximadamente un tercio de la longitud Stenoplax

3b. Longitud es aproximadamente el doble de la anchura. 4

4a. Área central de valvas intermedias con gruesa ornamentación en forma de red género Callistochiton, C. shuttleworthianus

4b. Diferente al anterior Ischnochiton

5a. Cinturón carnoso color amarillo marrón poblado de largas espículas quitinosas género Ceratozona, C. squalida

5b. Tegumento con superficie suave, a veces con gránulos microscópicos. Cinturón cubierto por corpúsculos calcáreos diminutos. Lepidochitona

\section{CLAVE PARA ESPECIES DE ISCHNOCHITON GRAY}

1a. Tegumento de todas las valvas finamente granulado. Áreas laterales de las valvas intermedias sin elevación, apenas se diferencia de las zonas centrales, tampoco están definidos los ápices ... I. papillosus

1b. Áreas laterales definidas, todas las valvas mostrando alguna escultura .2

2a. Ancho de las escamas dorsales del cinturón es mayor de $200 \mu \mathrm{m}$ I. striolatus

2b. Ancho de las escamas dorsales del cinturón es menor de $180 \mu \mathrm{m}$. I. erythronotus

CLAVE PARA ESPECIES DE STENOPLAX CARPENTER IN DALL (BASADO EN KAAS Y VAN BELLE, 1987)

1a. Valva anal más ancha que larga, mucrón en posición central

1b. Valva anal tan larga como ancha, mucrón en posición posterior S. bahamensis 
2a. valvas intermedias con estrías longitudinales en área lateral y central.

S. purpurascens

2b. Valvas intermedias con escultura longitudinal en áreas laterales y transversales y en áreas centrales, formando ángulo de 90 grados en la línea lateral

S. boogii

\section{CLAVE PARA ESPECIES DE LEPIDOCHITONA GRAY}

1a. Área lateral de las valvas intermedias distintamente levantadas. Valva anal con una mancha blanca en la región postmucronal

L. liozonis

1b. Área lateral de las valvas intermedias sin diferencia en altura

L. rosea

\section{CLAVE PARA GÉNERO Y ESPECIES DE CHITONIDAE RAFINESQUE}

1a. Cinturón dorsalmente cubierto por escamas

Chiton

1b. Cinturón cubierto con espinas calcáreas 2

2a.Tegumento de las valvas con superficie granulosa. Espinas del cinturón gruesas y cortas ..... Acanthopleuragranulata

2b.Tegumento con áreas centrales lisas. Cinturón dorsalmente cubierto con espículas calcáreas microscópicas, que da la impresión de estar desnudo. Tonicia schrammi

\section{CLAVE PARA ESPECIES DE CHITON LINNÉ}

1a. Valva sin escultura, lisa, generalmente pulida y brillosa. Áreas laterales poco definidas, apenas un poco levantadas Chiton marmoratus

1b. Valvas con escultura. 2

2a. Área central de las valvas sin ornamentación, excepto por rayones transversales, área lateral con 5-8 cordones granulosos en arreglo radial. Tegumento de color amarillo cobrizo con marcas irregulares color marrón. Chiton squamosus

2b. Área central con esculturas longitudinales 3

3a. Área central con 18 costillas redondeadas e irregulares, mejor definidas cerca del área lateral, esas costillas con apariencia de "S" invertida. Presencia de cuatro a cinco cordones con pocos pústulos en el área lateral. Chiton viridis

3b. Área central con surcos longitudinales que determinan la formación de costillas, valva cefálica y el área postmucronal de la valva anal están adornados con costillas nodulosas. Área lateral de las valvas intermedias levantadas con 5-8 costillas nodulosas irregulares . 


\section{CLAVE PARA GÉNEROS Y ESPECIES DE ACANTHOCHITONIDAE PILSBRY}

1a. Cinturón de apariencia carnosa, marrón oscuro a negro, cubre casi la totalidad de las valvas dejando descubierta sólo una banda estrecha del tegumento. Superficie del tegumento liso. Cinturón con 18 mechones de 10 espículas pequeñas y cortas. género Cryptoconchus, C. floridanus

1b. Cinturón del manto con amplia gama de colores, ancho y carnoso, cubierto de espículas vítreas dispersas por toda la superficie del cinturón. Valva cefálica mas ancha que larga, presencia de pústulos pequeños en el área lateropleural de las valvas intermedias. Acanthochitona

\section{CLAVE PARA ESPECIES DE ACANTHOCHITONA GRAY (BASADO EN LYONS, 1988)}

1a. Veinticinco o más espículas por mechón. 2

1b. Menos de 25 espículas por mechón 6

2a. Alrededor de 50 espículas por mechón 3

2b. Cien o más espículas por mechón 4

3a. Tegumento rojo oscuro con manchas blancas, cuerpo oval, pústulos redondeados, alrededor de 40-50 $\mu \mathrm{m}$ de diámetro A. hemphilli

3b. Cuerpo alargado, pústulos ovalados, $90 \mathrm{X} 45 \mu \mathrm{m}$ A. balesae

4a. Pústulos grandes, $120 \times 70 \mu \mathrm{m}$ A. pygmaea

4b. Pústulos con diámetros menores a $70 \mu \mathrm{m}$ .5

5a. Pústulos en forma de gota A. astrigera

5b. Pústulos redondos o un poco ovales A. lineata

6a. Margen del yugo de valvas intermedias ensancha hacia extremo anterior .7

6b. Yugo de valvas intermedias con márgenes paralelos A. roseojugum

7a. Color blanco con manchas negras, marrones, verdosas o rosadas A. andersoni

7b. Tres a 5 bandas color oliva o marrón, algunas valvas pueden ser de color oscuro. A. zebra 


\section{LITERATURA CITADA}

Aguayo, C. G. y M. L. Jaume. 1936. Sobre algunos moluscos marinos de Cuba. Memorias de la Sociedad Cubana de Historia Natural "Felipe Poey", 10, 115 - 122. En Espinosa, J. y J. Cruz de la.1985.Relaciones zoogeográficas de los quitones (Mollusca: Polyplacophora) de las Antillas. Paper presented at the Simposio de Ciencias del Mar y VII Jornada Científica del Instituto de Oceanología, La Habana.

Espinosa, J. 2006. Moluscos-Filo Mollusca. Lista de especies registradas en Cuba (octubre de 2006). En R. Claro ed., La Biodiversidad marina de Cuba. (CD-ROM), Instituto de Oceanología, Ministerio de Ciencia, Tecnología y Medio Ambiente, La Habana, Cuba.

Espinosa, J. y J. de la Cruz. 1985.Relaciones zoogeográficas de los quitones (Mollusca: Polyplacophora) de las Antillas. Paper presented at the Simposio de Ciencias del Mar y VII Jornada Científica del Instituto de Oceanología, La Habana.

Espinosa, J., R. Fernández-Garcés y E. Rolán. 1995. Catálogo actualizado de los moluscos marinos actuales de Cuba. Reseñas Malacológicas, 9: 1 - 90.

Espinosa, J., J. Ortea, R. Sánchez y J. Gutiérrez. 2012. Moluscos marinos Reserva de la Biosfera de la Península de Guanahacabibes. La Habana: Instituto de Oceanología. 325 pp. ilus. ISBN 978-959-298-024-2.

García-Ríos, C. 2003. Los quitones de Puerto Rico. Isla Negra, San Juan, 218 pp.

Kaas, P. 1972. Plyplacophora of the Caribbean Region. Studies on the fauna of Curaçao and others Caribbean Island.Vol. XLI.162 pp.

Kaas, P. y R. A. Van Belle. 1987. Monograph of living chitons (Mollusca: Polyplacophora), Vol. 3, Suborder Ischnochitonina: Ischnochitonidae: Chaetopleuridae, Ischnochitoninae (pars), additions to vols 1 y 2. E.J. Brill Publ., Leiden, Netherlands, 302 pp.

Kaas, P y R. A. Van Belle. 1998. Catalogue of living chitons (Mollusca, Polyplacophora). 2nda ed. Backhuys Publ., Leinden, Netherlands, 204 pp.

Lyons, W. G. 1988. A review of Caribbean Acanthochitonidae (Mollusca: Polyplacophora) with descriptions of six new species of Acanthochitona Gray, 1821. American Malacological Bulletin, 6: 79-114.

Lyons, W. G. y F. Moretzsohn. 2009. Polyplacophora (Mollusca) of the Gulf of Mexico. En J. W. y. F. Tunnell, D. L (Ed.), Gulf of Mexico origin, waters and biota. Vol. 1, Biodiversity, pp. $569-578$.

Slieker, F. J. A. 2000. Chitons of the World: an Illustrated Synopsis of Recent Polyplacophora. Ancona, Italy: L'Informatore Piceno.

[Recibido: 13 de mayo, 2015. Aceptado para publicación: 21 de agosto, 2015] 